\title{
Hyperbolic Harmonic Functions and Hyper- bolic Brownian Motion
}

\author{
Sirkka-Liisa Eriksson*®i and Terhi Kaarakka
}

\begin{abstract}
We study harmonic functions with respect to the Riemannian metric

$$
d s^{2}=\frac{d x_{1}^{2}+\cdots+d x_{n}^{2}}{x_{n}^{\frac{2 \alpha}{n-2}}}
$$

in the upper half space $\mathbb{R}_{+}^{n}=\left\{\left(x_{1}, \ldots, x_{n}\right) \in \mathbb{R}^{n}: x_{n}>0\right\}$. They are called $\alpha$-hyperbolic harmonic. An important result is that a function $f$ is $\alpha$-hyperbolic harmonic if and only if the function $g(x)=x_{n}^{-\frac{2-n+\alpha}{2}} f(x)$ is the eigenfunction of the hyperbolic Laplace operator $\triangle_{h}=x_{n}^{2} \triangle-(n-2) x_{n} \frac{\partial}{\partial x_{n}}$ corresponding to the eigenvalue $\frac{1}{4}\left((\alpha+1)^{2}-(n-1)^{2}\right)=0$. This means that in case $\alpha=n-2$, the $n$-2-hyperbolic harmonic functions are harmonic with respect to the hyperbolic metric of the Poincaré upper half-space. We are presenting some connections of $\alpha$-hyperbolic functions to the generalized hyperbolic Brownian motion. These results are similar as in case of harmonic functions with respect to usual Laplace and Brownian motion.
\end{abstract}

Mathematics Subject Classification. Primary 31EE, 60J45, Secondary 30 G35.

Keywords. Hyperbolic harmonic, Hyperbolic metric, Hyperbolic function theory, Brwonian motion, Hyperbolic Brownian motion.

\section{Introduction}

One of the major results in stochastics is that the classical potential theory, connected to Laplace equation, and theory of Brownian motion has strong relations found first by Kakutani [18] (see also [19]). We are pointing out similar results also between generalized Brownian motion and harmonic functions in

This article is part of the Topical Collection on ISAAC 12 at Aveiro, July 29-August 2, 2019, edited by Swanhild Bernstein, Uwe Kaehler, Irene Sabadini, and Franciscus Sommen.

${ }^{*}$ Corresponding author. 


$$
\mathbb{R}_{+}^{n}=\left\{\left(x_{0}, \ldots, x\right) \mid x_{1}, \ldots, x_{n} \in \mathbb{R}, x_{n}>0\right\}
$$

with respect to the Riemannian metric

$$
d s_{\alpha}^{2}=\frac{d x_{1}^{2}+\cdots+d x_{n}^{2}}{x_{n}^{\frac{2 \alpha}{n-2}}},
$$

where $\alpha \in \mathbb{R}$. The Riemannian metric $d s_{n-2}$ is the hyperbolic distance of the Poincaré upper half space. The Laplace-Beltrami operator connected to $d s_{\alpha}^{2}$ is

$$
\triangle_{\alpha} f=x_{n}^{\frac{2 \alpha}{n-2}}\left(\triangle f-\frac{\alpha}{x_{n}} \frac{\partial f}{\partial x_{n}}\right) .
$$

When $\alpha=n-2$ it is called the hyperbolic Laplace operator.

If a twice continuously differentiable function $f: \Omega \rightarrow \mathbb{R}$ satisfies $\triangle_{\alpha} f=$ 0 , it is called $\alpha$-hyperbolic harmonic. If $\alpha=n-2$, then $\alpha$-hyperbolic harmonic functions are called briefly hyperbolic harmonic.

We recall the result.

Theorem 1.1. [20, Lemma 2.1] Let $\Omega$ be an open set contained in $\mathbb{R}_{+}^{n+1}$. A twice continuously differentiable function $f: \Omega \rightarrow \mathbb{R}$ is $\alpha$-hyperbolic harmonic if and only if the function $g(x)=x_{n^{\frac{n-2-\alpha}{2}}} f(x)$ is a solution of the equation

$$
\triangle_{n-2} g+\frac{1}{4}\left((n-1)^{2}-(\alpha+1)^{2}\right) g=0 .
$$

The hyperbolic distance $d_{h}(x, y)$ between the points $x$ and $y$ in $\mathbb{R}_{+}^{n+1}$ may be computed as follows

$$
\begin{aligned}
d_{h}(x, y) & =\inf _{\gamma(0)=x, \gamma(1)=y} \int_{\gamma} \frac{\sqrt{\gamma_{1}^{\prime 2}(t)+\cdots+\gamma_{n}^{\prime 2}(t)}}{\gamma_{n}(t)} d t \\
& =\ln \left(\lambda(x, y)+\sqrt{\lambda(x, y)^{2}-1}\right)
\end{aligned}
$$

where $\lambda(x, y)=1+\frac{|x-y|^{2}}{2 x_{n} y_{n}}$ and $\cosh d_{h}(x, y)=\lambda(x, y)$.

The geodesics, representing the shortest distance between the points, are circular arcs perpendicular to the hyperplane $x_{n}=0$ (that is, half-circles whose origin is on $x_{n}=0$ and straight vertical lines ending on the hyperplane $\left.x_{n}=0\right)$. $[22])$.

We recall important properties of hyperbolic distance (see for example

Lemma 1.2. The hyperbolic ball $D_{h}\left(a, r_{h}\right)$ in $\mathbb{R}_{+}^{n+1}$ with the hyperbolic center $a=\left(a_{1}, . ., a_{n}\right)$ and the radius $r_{h}$ is the same as the Euclidean ball $D_{e}\left(c_{a}\left(r_{h}\right), r_{e}\right)$ with the center

$$
c_{a}\left(r_{h}\right)=\left(a_{1}, \ldots, a_{n} \cosh r_{h}\right)
$$

and the Euclidean radius $r_{e}=a_{n} \sinh r_{n}$.

The hyperbolic distance has the following invariance property. 
Theorem 1.3. The group under composition of orientation preserving Möbius transformations mapping the upper half space bijectively onto itself is the group of isometries of the hyperbolic upper half space model, that is mappings $f$ satisfying

$$
d_{h}(f(v), f(w))=d_{h}(v, w)
$$

(that is, the orientation preserving transformations mapping the upper half space onto itself are translations, dilatations, special orthogonal transformations and the inversion with respect to the sphere mapping the upper half bijectively onto itself and their compositions).

Moreover, hyperbolic harmonic functions are invariant under orientation preserving Möbius transformations.

Theorem 1.4. Let $\Omega$ be an open subset of the upper half space $\mathbb{R}_{+}^{n}$. If $f: \Omega \rightarrow$ $\mathbb{R}$ is hyperbolic harmonic then $f \circ T$ is also hyperbolic harmonic on $T^{-1}(\Omega)$ for any orientation preserving Möbius transformation $T$.

We recall the definition of the $n$-dimensional Brownian motion.

Definition 1.5. Let $(\Omega, \mathcal{F}, \mathbb{P})$ be a probability space and $\left\{B_{t}\right\}_{t>0}$ be an $\mathbb{R}^{n}$ valued stochastic process. Then $\left\{B_{t}\right\}_{t \geq 0}$ is called a standard $n$-dimensional Brownian motion, if the following properties hold:

(i) $B_{0}=0$;

(ii) The function $t \rightarrow B_{t}$ is continuous almost surely;

(iii) The process $\left\{B_{t}\right\}_{t \geq 0}$ has independent increments, that is $B_{t+s}-B_{s}$ is independent of $\left(B_{s_{1}}, \ldots, B_{s_{k}}\right)$ for all $0 \leq s_{1} \leq s_{2} \leq \cdots \leq s_{k} \leq s<$ $s+t<\infty$

(iv) The increments are stationary, that is, $B_{t+s}-B_{s}$ has $n$-normal distribution with the mean 0 and the covariance $t I$, that is,

$$
\begin{aligned}
P\left(B_{t+s}-B_{s} \in E\right) & =P\left(B_{t} \in E\right) \\
& =\int_{E}(2 \pi t)^{-\frac{n}{2}} \exp \left(-\frac{\|x\|^{2}}{2 t}\right) d x .
\end{aligned}
$$

An $\mathbb{R}^{n}$-valued continuous-time stochastic process $\left\{B_{t}^{u}\right\}_{t>0}$ is called an $n$-dimensional Brownian motion started at $u$ if the process $\left\{\bar{B}_{t}^{u}-u\right\}_{t \geq 0}$ is a standard $n$-dimensional Brownian motion. We denote the transition probability $P_{u}^{t}$ by

$$
P_{u}^{t}\left(B_{t}^{u} \in E\right)=P\left(B_{t}^{u} \in E\right) .
$$

From the definition we obtain directly that the Radon-Nikodym derivative is

$$
\frac{d P_{u}^{t}}{d x}=(2 \pi t)^{-\frac{n}{2}} \exp \left(-\frac{\|x-u\|^{2}}{2 t}\right)
$$




\section{Harmonic Measure}

Solutions of a general class of elliptic equations behave in a similar way as harmonic functions with respect to the Laplace equation. In order to use these type of results, we first recall a general definition of Brelot space, introduced by Brelot in [5].

Definition 2.1. Let $X$ be a locally compact Hausdorff space. A mapping $\mathcal{H}$ : $U \rightarrow \mathcal{H}(U)$ from an open subset of $X$ is called a harmonic sheaf if for any open subset $U$ of $X$ the set $\mathcal{H}(U)$ is a vector subspace on real continuous functions on $U$. A function $h: W \rightarrow \mathbb{R}$ from an open subset $W$ of $X$ is called harmonic if its restriction to any open subset $U$ of $W$ belong to $\mathcal{H}(U)$.

A relatively compact open set $U \subset X$ is called regular if for any continuous $f \in \mathcal{C}(\partial U)$ there exists a harmonic function $h$ on $U$ such that it has a continuous extension $\bar{h} \in \mathcal{C}(\bar{U})$ satisfying

$$
f=\bar{h}_{\mid \partial U} \text {. }
$$

The pair $(X, \mathcal{H})$ is called a Brelot space, if $X$ is locally connected and does not contain any isolated points and the following properties hold:

(a). For any point $x \in X$ there exists a harmonic function $h \in \mathcal{H}(U)$ for some open neighborhood $U$ of $x$ and $h(x) \neq 0$, (that is $\mathcal{H}$ non-generate at any point $x \in X)$;

(b). The harmonic sheaf $\mathcal{H}$ satisfies the Brelot convergence property. That is, the limit of any increasing sequence of harmonic functions on a connected open set is harmonic provided it is finite at a point;

(c). Regular sets form a basis for the topology on $X$.

Brelot spaces, introduced by Brelot in 1957, are also harmonic spaces defined by Constantinescu and Cornea [10].

Theorem 2.2. Let $U \subset X$ be regular. Then for any point $x \in U$ there exists a (Radon) measure $L_{x}^{U}: f \rightarrow H_{f}^{U}(x)$ such that for any continuous $f \in \mathcal{C}(\partial U)$ and for any $x \in U$, the function $H_{f}^{U}$ is harmonic in $U$ and $\lim _{x \rightarrow y} H_{f}^{U}(x)=$ $f(y)$ for any $y \in \partial U$. A Radon measure induces a regular Borel measure $\mu_{x}^{U}$ on $\partial U$ such that

$$
\int_{\partial U} f d \mu_{x}^{U}=H_{f}^{U}(x)
$$

for any continuous $f \in \mathcal{C}(\partial U)$. If a function $f \equiv 1$ is harmonic, then the harmonic measure $\mu_{x}^{U}$ is a probability measure on $\partial U$.

Theorem 2.3. If $\mathcal{H}(U)$ is a set of $\alpha$-hyperbolic harmonic functions on open subset $U$ of $\mathbb{R}_{+}^{n}$, then $\mathcal{H}: U \rightarrow \mathcal{H}(U)$ is a harmonic sheaf and the pair $\left(\mathbb{R}_{+}^{n}, \mathcal{H}\right)$ is a Brelot space, where the regular sets are the same as with respect to the usual Laplace equation. Moreover, the harmonic measure $\mu_{x}^{U}$ is a probability measure on $\partial U$ for any regular set $U \subset \mathbb{R}_{+}^{n}$.

This result is a special case of the theorem concerning elliptic differential equations, proved for example by R.-M. Herve. It is stated in [10, p. 79] with several references. 
An important tool for computing the harmonic measure of the Laplace operator is the fundamental solution (see [17]). Applying [12] or [13], we find the fundamental $\alpha$-hyperbolic function from the function

$$
\left(\lambda^{2}-1\right)^{\frac{1-n}{4}} Q_{\frac{|k+1|-1}{2}}^{ \pm \frac{n-1}{2}}(\lambda),
$$

where $Q_{\frac{|k+1|-1}{2}}^{ \pm \frac{n-1}{2}}(\lambda)$ is the Legendre function of the second kind, defined in $[16,8.703]$ by

$$
Q_{\nu}^{\beta}(u)=C\left(u^{2}-1\right)^{\frac{\beta}{2}} u^{-\nu-\beta-1}{ }_{2} F_{1}\left(\frac{\beta+\nu+2}{2}, \frac{\beta+\nu+1}{2} ; \frac{2 \nu+3}{2} ; \frac{1}{u^{2}}\right),
$$

where

$$
C=\frac{\sqrt{\pi} \Gamma(\beta+\nu+1) e^{i \beta \pi}}{2^{\nu+1} \Gamma\left(\nu+\frac{3}{2}\right)}
$$

and ${ }_{2} F_{1}$ is the hypergeometric function

$$
{ }_{2} F_{1}(a, b ; c ; x)=\sum_{m=0}^{\infty} \frac{(a)_{m}(b)_{m}}{(c)_{m}} \frac{x^{m}}{m !},
$$

converging for $x$ satisfying $|x|<1$. We recall the Euler relation

$$
{ }_{2} F_{1}(a, b, c ; x)=(1-x)^{c-a-b}{ }_{2} F_{1}(c-a, c-b, c ; x)
$$

and the equality

$$
\begin{aligned}
{ }_{2} F_{1}\left(\frac{\nu-\beta+2}{2}, \frac{\nu-\beta+1}{2} ; \frac{2 \nu+3}{2} ; 1\right) & =\frac{\Gamma\left(\nu+\frac{3}{2}\right) \Gamma(\beta)}{\Gamma\left(\frac{\nu+\beta+1}{2}\right) \Gamma\left(\frac{\nu+\beta+2}{2}\right)} \\
& =\frac{\Gamma\left(\nu+\frac{3}{2}\right) \Gamma(\beta) 2^{\nu+\beta}}{\sqrt{\pi} \Gamma(\nu+\beta+1)} .
\end{aligned}
$$

(see [2, Theorem 2.2.2. and Theorem 2.2.5] and [16, 8.335]). Setting $u=$ $\cosh r_{h}=\lambda(x, y)$, we denote

$$
s_{\beta, \nu}\left(r_{h}\right)=C_{\beta, \nu} \cosh ^{\beta-\nu-1} r_{h 2} F_{1}\left(\frac{\nu-\beta+2}{2}, \frac{\nu-\beta+1}{2} ; \frac{2 \nu+3}{2} ; \frac{1}{\cosh ^{2} r_{h}}\right) \text {, }
$$

where

$$
C_{\beta, \nu}=\frac{\sqrt{\pi} \Gamma(\beta+\nu+1)}{2^{\beta-1} \Gamma(\beta) \Gamma\left(\nu+\frac{3}{2}\right)} .
$$

Then $s_{\beta, \nu}(0)=1$. The surface measure $\omega_{n}$ of the Euclidean unit ball is

$$
\omega_{n}=\frac{\pi^{\frac{n}{2}} n}{\Gamma\left(\frac{n+2}{2}\right)} .
$$

The fundamental $\alpha$-hyperbolic harmonic function was found in [12], but it included constants which were not computed. We verify that the function

$$
H_{\alpha}(x, y)=\frac{x_{n}^{\frac{\alpha+2-n}{2}} y_{n}^{\frac{\alpha+2-n}{2}} s_{\frac{n-2}{2}, \frac{\alpha}{2}}\left(r_{h}\right)}{\omega_{n}(n-2) \sinh ^{n-2}\left(r_{h}\right)}
$$


is the fundamental $\alpha$-hyperbolic harmonic function. The function $H_{\alpha}(x, y)$ has the different coefficient than the function computed in [13].

We first notice that if $y \in \mathbb{R}_{+}^{n+1}$ is fixed the function $H_{\alpha}(x, y)$ is $\alpha$ hyperbolic harmonic with respect to the variable $x$ if $x \neq y$ (that is $d_{h}(x, y) \neq$ $0)$. Similarly, if $x \in \mathbb{R}_{+}^{n+1}$ is fixed it is $\alpha$-hyperbolic harmonic with respect to the variable $y$ if $y \neq x$. It is also Lebesgue integrable in the hyperbolic ball $D_{h}\left(x, R_{h}\right)$.

Lemma 2.4. The function $H_{\alpha}(x, y)$ is Lebesgue integrable in the hyperbolic ball $D_{h}\left(x, R_{h}\right)$ and

$$
\int_{D_{h}\left(x, R_{h}\right)}\left|H_{\alpha}(x, y)\left(d_{h}(x, y)\right)\right| \frac{d m(y)}{y_{n}^{\frac{\alpha(n-1)}{n-2}}} \leq M\left(x, R_{h}\right) \sinh ^{2} R_{h}
$$

for some positive constant $M\left(x, R_{h}\right)>0$.

The proof is similar as in [12].

Lemma 2.5. Let $\Omega \subset \mathbb{R}_{+}^{n+1}$ be open. Let $u$ be continuous in $\Omega$. Then

$$
\lim _{R_{h} \rightarrow 0} \int_{\partial D_{h}\left(x, R_{h}\right)} u \frac{\partial H_{\alpha}(x, y)}{\partial m_{(\alpha)}} d \sigma_{(\alpha)}(y)=-u(x)
$$

for any hyperbolic balls $D_{h}\left(x, R_{h}\right)$ satisfying $\overline{D_{h}\left(x, R_{h}\right)} \subset \Omega$, where

$$
d \sigma_{(\alpha)}(y)=y_{n}^{-\frac{\alpha(n-1)}{n-2}} d \sigma(y), \frac{\partial}{\partial m_{(\alpha)}}=y_{n}^{\frac{\alpha}{n-2}} \frac{\partial}{\partial m_{e}},
$$

$m_{e}$ the outward-pointing normal of $D_{h}\left(x, R_{h}\right)$ and $\omega_{n}$ is the surface measure of the unit ball.

Proof. Lemma 1.2 implies that the outward-pointing normal at $y \in$ $\partial D_{h}\left(x, R_{h}\right)$ is given by

$$
m_{e}=\left(m_{1}, \ldots, m_{n}\right)=\frac{\left(y_{1}-x_{1}, \ldots, y_{n}-x_{n} \cosh R_{h}\right)}{x_{n} \sinh R_{h}} .
$$

Set $r_{h}=d_{h}(x, y)$. Since $\cosh r_{h}=\lambda(y, x)$ we compute

$$
\frac{\partial r_{h}}{\partial y_{i}}=\frac{\partial r_{h}}{\partial y_{i}}=\frac{\partial \arccos \lambda(x, y)}{\partial y_{i}}=\frac{y_{i}-x_{i}-x_{n}\left(\cosh r_{h}-1\right) \delta_{n}(i)}{y_{n} x_{n} \sinh r_{h}}
$$

and

$$
\sum_{i=1}^{n} m_{i} \frac{\partial r_{h}}{\partial y_{i}}=\frac{1}{y_{n}}
$$

Set

$$
\gamma(\alpha, n)=\frac{(n-2)(\alpha-n)+2 \alpha}{2(n-2)} .
$$

Hence we deduce that

$$
\frac{\partial H_{\alpha}(x, y)}{\partial m_{(\alpha)}}=y_{n}^{\gamma(\alpha, n)} x_{n}^{\frac{\alpha+2-n}{2}} \frac{s_{\frac{n-2}{2}, \frac{\alpha}{2}}^{\prime}\left(r_{h}\right)}{\sinh ^{n-2} r_{h}}
$$




$$
\begin{aligned}
& -(n-2) y_{n}^{\gamma(\alpha, n)} x_{n}^{\frac{\alpha+2-n}{2}} \frac{s_{\frac{n-2}{2}, \frac{\alpha}{2}} \cosh r_{h}}{\sinh ^{n-1} r_{h}} \\
& +\frac{\alpha+2-n}{2} y_{n}^{\gamma(\alpha, n)} x_{n}^{\frac{\alpha+2-n}{2}} m_{n} \frac{s_{\frac{n-2}{2}}, \frac{\alpha}{2}\left(r_{h}\right)}{\sinh ^{n-2} r_{h}} .
\end{aligned}
$$

Using Lemma 1.2 we infer that $D_{h}\left(x, R_{h}\right)=D_{e}\left(x_{e}, x_{n} \sin R_{h}\right)$ and $x_{e}=$ $\left(x_{0}, x_{1}, \ldots, x_{n} \cosh R_{h}\right)$, which implies

$$
\lim _{R_{h} \rightarrow 0} \frac{x_{n}^{\frac{\alpha+n}{2}} \sinh R_{h}}{\omega_{n} x_{n}^{n-1} \sinh ^{n-1} R_{h}} \int_{\partial D_{h}\left(x, R_{h}\right)} y_{h}^{\gamma(\alpha, n)} u(y) s_{\frac{n-2}{2}, \frac{\alpha}{2}}^{\prime}\left(R_{h}\right) d \sigma_{(\alpha)}=0,
$$

since $\sinh R_{h}$ goes to 0 and the rest of the formula is just the average value of the continuous function over $D_{h}\left(x, R_{h}\right)$. Similarly, we compute that

$$
\lim _{R_{h} \rightarrow 0} \frac{x_{n}^{\frac{\alpha+n}{2}} \sinh R_{h}}{\omega_{n} x_{n}^{n-1} \sinh ^{n-1} R_{h}} \int_{\partial D_{h}\left(x, R_{h}\right)} y_{n}^{\gamma(\alpha, n)} m_{n} s_{\frac{n-2}{2}, \frac{\alpha}{2}}\left(r_{h}\right) u(y) d \sigma_{(\alpha)}=0 .
$$

Since $d \sigma_{(\alpha)}(y)=y_{n}^{-\frac{\alpha(n-1)}{n-2}} d \sigma(y)$, we obtain in the last integral

$$
\begin{aligned}
& \lim _{R_{h} \rightarrow 0}-\frac{x_{n}^{\frac{\alpha+n}{2}} \cosh R_{h}}{\omega_{n} x_{n}^{n-1} \sinh ^{n-1} R_{h}} \int_{\partial D_{h}\left(x, R_{h}\right)} y_{n}^{\gamma(\alpha, n)} s_{\frac{n-2}{2}, \frac{\alpha}{2}}\left(r_{h}\right) u(y) d \sigma_{(\alpha)} \\
& \quad=\lim _{R_{h} \rightarrow 0}-\frac{x_{n}^{\frac{\alpha+n}{2}} \cosh R_{h}}{\omega_{n} x_{n}^{n-1} \sinh ^{n-1} R_{h}} \int_{\partial D_{h}\left(x, R_{h}\right)} u(y) \frac{s_{\frac{n-2}{2}, \frac{\alpha}{2}}\left(r_{h}\right)}{y_{n}^{\frac{\alpha+n}{2}}} d \sigma \\
& =-u(x),
\end{aligned}
$$

completing the proof.

Theorem 2.6. Let $\Omega \subset \mathbb{R}_{+}^{n+1}$ be open and $D_{h}(a, \rho)$ a hyperbolic ball with a center $a$ and the hyperbolic radius $\rho$ satisfying $\overline{B_{h}(a, \rho)} \subset \Omega$. If $u$ is a twice continuously differentiable function in $\Omega$ and $x \in D_{h}(a, \rho)$, then

$$
\begin{aligned}
u(x)= & \int_{\partial D_{h}(a, \rho)}\left(H_{\alpha}(x, y) \frac{\partial u(y)}{\partial m_{(\alpha)}}-u(y) \frac{\partial H_{\alpha}(x, y)}{\partial m_{(\alpha)}}\right) d \sigma_{(\alpha)}(y) \\
& -\int_{D_{h}(a, \rho)} \triangle_{\alpha} u H_{\alpha}(x, y) d y_{(\alpha)},
\end{aligned}
$$

where $d y_{(\alpha)}=y_{n}^{-\frac{\alpha n}{n-2}} d y, d \sigma_{(\alpha)}=y_{n}^{-\frac{\alpha(n-1)}{n-2}} d \sigma$ and $\frac{\partial u}{\partial m_{(\alpha)}}=y_{n}^{\frac{\alpha}{n-2}} \frac{\partial u}{\partial m_{e}}$.

Proof. Denote $D_{h}(a, \rho)=D$ and pick a hyperbolic ball such that $\overline{D_{h}\left(x, R_{h}\right)} \subset$ $D$. Set $R=D \backslash D_{h}\left(x, R_{h}\right)$. Since $H_{\alpha}$ is $\alpha$-hyperbolic harmonic, applying the Green's identity

$$
\int_{R}\left(u \triangle_{\alpha} v-v \triangle_{\alpha} u\right) d y_{(\alpha)}=\int_{\partial R}\left(u \frac{\partial v}{\partial m_{(\alpha)}}-v \frac{\partial u}{\partial m_{(\alpha)}}\right) d \sigma_{(\alpha)}
$$

of the Laplace-Beltrami operator

$$
\triangle_{\alpha}=y_{n}^{\frac{2 \alpha}{n-2}}\left(\triangle-\frac{\alpha}{y_{n}} \frac{\partial}{\partial y_{n}}\right)
$$


with respect to the Riemannian metric $d s_{\alpha}^{2}$ (see [1]) we obtain

$$
\begin{aligned}
\int_{R} H_{\alpha}(x, y) \triangle_{\alpha} u d y_{(\alpha)}= & \int_{\partial D}\left(H_{\alpha}(x, y) \frac{\partial u}{\partial m_{(\alpha)}}-u \frac{\partial H_{\alpha}(x, y)}{\partial m_{(\alpha)}}\right) d \sigma_{(\alpha)} \\
& -\int_{\partial D_{h}\left(x, R_{h}\right)}\left(H_{\alpha}(x, y) \frac{\partial u}{\partial m_{(\alpha)}}-u \frac{\partial H_{\alpha}}{\partial m_{(\alpha)}}(x, y)\right) d \sigma_{(\alpha)} .
\end{aligned}
$$

Since

$$
\int_{\partial D_{h}\left(x, R_{h}\right)}\left|H_{\alpha}(x, y) \frac{\partial u}{\partial m_{(\alpha)}}\right| d \sigma_{(\alpha)}(y) \leq \frac{M \sinh R_{h}}{\omega_{n} \sinh ^{n-1} R_{h}} \int_{\partial D_{h}\left(x, R_{h}\right)} d \sigma
$$

and $\sinh R_{h}$ goes to 0 we infer that the rest of the formula is just the average value of the continuous function over $D_{h}\left(x, R_{h}\right)$ and we conclude

$$
\lim _{R_{h} \rightarrow 0} \int_{\partial D_{h}\left(x, R_{h}\right)}\left|H_{\alpha}(x, y) \frac{\partial u}{\partial m_{(\alpha)}}\right| d \sigma_{(\alpha)}(y)=0 .
$$

Applying the previous result we obtain the desired result.

In case $\phi \in \mathcal{C}_{0}^{\infty}\left(\mathbb{R}_{+}^{n+1}\right)$ we obtain

$$
\phi(x)=-\int \triangle_{\alpha} \phi(y) H_{\alpha}(x, y) d y_{(\alpha)}
$$

and therefore $-\triangle_{\alpha} H_{\alpha}(x, y)=\delta_{x}$ in the distribution sense. We conclude the following result.

Theorem 2.7. The function $H_{\alpha}(x, y)$ is the fundamental $\alpha$-hyperbolic harmonic function.

The fundamental solution may also be computed using hyperbolic Brownian motion explained in the next section.

\section{Hyperbolic Brownian Motion}

Heinz Leutwiler started in 1987 [20] to study potential theoretic properties of solutions of the Weinstein equation

$$
\triangle f-\frac{k}{x_{n}} \frac{\partial f}{\partial x_{n}}+l \frac{f}{x_{n}^{2}}=0 .
$$

Later hyperbolic function theory was initiated by Heinz Leutwiler around $1990[21,22]$ and further developed together with the first author (see [11]).

Hyperbolic Brownian motion was defined in 1996 by Gruet [15]. Several persons have been researching it: Baldi et al. [3,4], Byczkowski et al. [7-9], Małecki and Serafin [23], Franchi and Le Jan [14]. We define a $\alpha$-hyperbolic Brownian motion, called also a hyperbolic Brownian motion with drift $\mu=$ $\frac{\alpha+1}{2}$ (see [6] and [23]).

Definition 3.1. Let $\alpha \geq 1$. A $\alpha$-hyperbolic Brownian motion is a unique strong solution $X(t)=\left(X_{1}(t), \ldots, X_{n}(t)\right)$ corresponding to the system of stochastic differential equations

$$
d X_{i}(t)=X_{n}(t) d B_{i}, i=1, \ldots, n-1,
$$




$$
\begin{aligned}
d X_{n}(t) & =X_{n}(t) d B_{n}-\frac{\alpha}{2} X_{n}(t) d t, \\
X(0) & =x \in \mathbb{R}_{+}^{n}, \\
E B_{i}^{2}(t) & =t \text { for } i=1, \ldots, n,
\end{aligned}
$$

where $B_{1}, \ldots, B_{n}$ are independent standard Brownian motions in $\mathbb{R}$.

The generator of this diffusion is given by

$$
\lim _{t \rightarrow 0} \frac{E_{x}(f(X(t)))-f(x)}{t}=\frac{1}{2}\left(x_{n}^{2} \triangle f-\alpha x_{n} \frac{\partial f}{\partial x_{n}}\right),
$$

if $f: \mathbb{R}^{n+1} \rightarrow \mathbb{R}$ and $f \in C^{2}\left(\mathbb{R}^{n+1}\right)$.

We also immediately notice that the hyperbolic Brownian motion is $n$-2- hyperbolic Brownian motion. Using Itô calculus, we obtain the solution.

Proposition 3.2. Let $\mu=\frac{\alpha+1}{2}$. The solution $X^{(\mu)}(t)$ of the previous system of stochastic differential equations is generated by the geometric Brownian motion $X_{n}^{(\mu)}(t)$ with drift $-\mu$

$$
X_{n}^{(\mu)}(t)=x_{n} \exp \left(B_{n}(t)-\mu t\right)
$$

and

$$
\begin{aligned}
X_{1}^{(\mu)}(t) & =x_{1}+\int_{0}^{t} X_{n}^{(\mu)}(t) d B_{1}(u), \\
& \vdots \\
X_{n-1}^{(\mu)}(t) & =x_{n-1}+\int_{0}^{t} X_{n}^{(\mu)}(t) d B_{n-1}(u),
\end{aligned}
$$

where $B_{1}, \ldots, B_{n}$ are standard Brownian motions. $[23])$.

$\alpha$-hyperbolic Brownian motion has the following characterization (see

Proposition 3.3. Let $B=\left(B_{1}, \ldots, B_{n-1},\right)$ be a standard Brownian motion $\mathbb{R}^{n-1}$ independent of the geometric Brownian motion $X_{n}^{(\mu)}$ with drift $-\mu$. Denote

$$
A_{x_{n}}^{(-\mu)}(t)=x_{n} \int_{0}^{t} \exp \left(2 X_{n}^{(-\mu)}(u)\right) d u .
$$

Then $2 \mu-1$-hyperbolic Brownian motion $X^{(\mu)}$ generated by the geometric Brownian motion $X_{n}^{(\mu)}$ with drift $-\mu$ has the property

$$
X^{(\mu)}(t) \stackrel{d}{=} Y^{(\mu)}\left(A_{x_{n}}^{(-\mu)}(t)\right)
$$

where

$$
Y^{(\mu)}(t)=\left(B_{1}(t), \ldots, B_{n-1}(t), R^{(-\mu)}(t)\right)
$$

and $R^{(-\mu)}=\left\{R_{t}^{(-\mu)} \mid t \geq 0\right\}$ is a Bessel motion with the index $-\mu$ starting from $x_{n}>0$ independent from the Brownian motion $B$.

Using the previous result, the transition density function of the generalized hyperbolic Brownian motion was computed by Małecki and Serafin in $[23]$. 
Theorem 3.4. Let $\mu=\frac{\alpha+1}{2} \geq 0$. The transition density function of $X^{(\mu)}$ with respect to the hyperbolic volume element $x_{n}^{-n} d x$ is

$$
p^{(\mu)}(t, x, y)=\left(\frac{x_{n}}{y_{n}}\right)^{\mu-\frac{n-1}{2}} \frac{e^{-\frac{\mu^{2} t}{2}} \Gamma\left(\frac{n+1}{2}\right)}{\pi(2 \pi)^{\frac{n}{2}} t^{\frac{1}{2}}} \int_{0}^{\infty} \frac{e^{\frac{\pi^{2}-u^{2}}{2 t}} \sinh u \sin \left(\frac{\pi u}{t}\right)}{(\cosh u+\cosh \rho)^{\frac{n+1}{2}}} d u,
$$

where $\rho=d_{h}(x, y)$.

Our main result is the following connection between the potential kernel and the fundamental solution.

Theorem 3.5. Let $\mu=\frac{\alpha+1}{2} \geq 0$ and

$$
X^{(\mu)}(t)=\left(X_{1}^{(\mu)}(t), \ldots, X_{n}^{(\mu)}(t)\right)
$$

be the $\alpha$-hyperbolic Brownian motion. Then the potential kernel $V^{(\mu)}(x, y)$ defined in [23] by

$$
V^{(\mu)}(x, y)=\int_{0}^{\infty} p^{(\mu)}(t, x, y) d t
$$

satisfies the equality

$$
y_{n}^{\alpha+2-n} V^{(\mu)}(x, y)=2 H_{\alpha}\left(x_{n}, y_{n}\right) .
$$

Proof. Set $\beta=\frac{n-2}{2}$ and $\nu=\frac{\alpha}{2}$. We infer from [23] that

$$
V^{\left(\frac{\alpha+1}{2}\right)}(x, y)=\frac{x_{n}^{\nu-\beta}}{y_{n}^{\nu-\beta}} \frac{2 e^{-\beta i \pi} Q_{\nu}^{\beta}\left(\cosh r_{h}\right)}{(2 \pi)^{\frac{n}{2}} \sinh ^{\beta}\left(r_{h}\right)} .
$$

Applying (2.1) we obtain

$$
V^{\left(\frac{\alpha+1}{2}\right)}(x, y)=\frac{x_{n}^{\nu-\beta}}{y_{n}^{\nu-\beta}} \frac{2 C_{0}\left(u^{2}-1\right)^{\frac{\beta}{2}}{ }_{2} F_{1}\left(\frac{\beta+\nu+2}{2}, \frac{\beta+\nu+1}{2} ; \frac{2 \nu+3}{2} ; \frac{1}{u^{2}}\right)}{u^{\beta+\nu+1}(2 \pi)^{\frac{n}{2}} \sinh ^{\beta}\left(r_{h}\right)},
$$

where $u=\cosh r_{h}$ and

$$
C_{0}=\frac{\sqrt{\pi} \Gamma(\beta+\nu+1)}{2^{\nu+1} \Gamma\left(\nu+\frac{3}{2}\right)} .
$$

Using the Euler relation and (2.2) we obtain

$$
\begin{aligned}
V^{\left(\frac{\alpha+1}{2}\right)}(x, y) & =\frac{x_{n}^{\nu-\beta}}{y_{n}^{\nu-\beta}} \frac{2 C_{0}{ }_{2} F_{1}\left(\frac{\nu-\beta+2}{2}, \frac{\nu-\beta+1}{2} ; \frac{2 \nu+3}{2} ; \frac{1}{u^{2}}\right)}{u^{\nu+1-\beta} \sinh ^{n-2}\left(r_{h}\right)} \\
& =\frac{2 s_{\beta, \nu}\left(r_{h}\right) \Gamma(\beta)}{\sinh ^{n-2}\left(r_{h}\right) 4 \pi^{\frac{n}{2}} y_{n}^{2(\nu-\beta)}} .
\end{aligned}
$$

Applying $\Gamma(x+1)=x \Gamma(x)$ and

$$
\begin{aligned}
\omega_{n} & =\frac{\pi^{\frac{n}{2}} n}{\Gamma\left(\frac{n+2}{2}\right)}=\frac{2 \pi^{\frac{n}{2}}}{\Gamma\left(\frac{n}{2}\right)} \\
& =\frac{4 \pi^{\frac{n}{2}}}{(n-2) \Gamma\left(\frac{n-2}{2}\right)}=\frac{4 \pi^{\frac{n}{2}}}{(n-2) \Gamma(\beta)}
\end{aligned}
$$


we conclude

$$
y_{n}^{\alpha+2-n} V^{\left(\frac{\alpha+1}{2}\right)}(x, y)=2 H_{a}(x, y) .
$$

We recall the definition of the first exit time from a set $D$ (the first hit time for the complement of $D$ ).

Definition 3.6. If $D$ is a nonempty, open, connected bounded subset of $\mathbb{R}^{n}$, then we denote

$$
\tau_{D}=\left\{\begin{array}{l}
\inf \left\{t>0: X_{t} \notin D\right\}, \\
\infty, \text { if there is no such } t>0 .
\end{array}\right.
$$

We recall the Dynkin formula and its direct corollary.

Theorem 3.7. (Dynkin formula) Let $f: \bar{D} \rightarrow \mathbb{R}$ be a bounded, continuous function defined on the closure of $D$. If $f$ has bounded first and second partial derivatives in $D$, then for every $x \in D$,

$$
E^{x} f\left(X_{\tau_{D}}\right)=f(x)+\frac{1}{2} E^{x} \int_{0}^{\tau_{D}} \triangle_{\alpha} f\left(X_{s}\right) d s .
$$

Moreover, if $h$ is $\alpha$-hyperbolic harmonic in $\Omega$ and $\bar{D} \subset \Omega$, then

$$
E^{x} h\left(X_{\tau_{D}}\right)=h(x) .
$$

In case of harmonic functions with respect to Laplace operator, Kakutani [18] (see also [19]) noticed that the harmonic measure has a probabilistic interpretation. If $K$ is a nice compact subset of $\partial U$, then the harmonic measure $\mu_{x}^{U}(K)$ is also equal to the probability that a Brownian motion started at $x$ reaches $K$ before hitting $\partial U \backslash K$. Future problem is to find out if the similar result holds for $\alpha$-hyperbolic Brownian motion. The difficulty is the computation of the harmonic measure of $\alpha$-hyperbolic harmonic functions.

Funding Open access funding provided by University of Helsinki including Helsinki University Central Hospital.

Open Access. This article is licensed under a Creative Commons Attribution 4.0 International License, which permits use, sharing, adaptation, distribution and reproduction in any medium or format, as long as you give appropriate credit to the original author(s) and the source, provide a link to the Creative Commons licence, and indicate if changes were made. The images or other third party material in this article are included in the article's Creative Commons licence, unless indicated otherwise in a credit line to the material. If material is not included in the article's Creative Commons licence and your intended use is not permitted by statutory regulation or exceeds the permitted use, you will need to obtain permission directly from the copyright holder. To view a copy of this licence, visit http:// creativecommons.org/licenses/by/4.0/.

Publisher's Note Springer Nature remains neutral with regard to jurisdictional claims in published maps and institutional affiliations. 


\section{References}

[1] Akin, Ö., Leutwiler, H.: On the invariance of the solutions of the Weinstein equation under Möbius transformations. In: Classical and modern potential theory and applications (Chateau de Bonas, 1993), 19-29, NATO Adv. Sci. Inst. Ser. C Math. Phys. Sci., 430, Kluwer, Dordrecht (1994)

[2] Andrews, G.E., Askey, R., Roy, R.: Special Functions. Cambridge University Press, Cambridge (1999)

[3] Baldi, P., Casadio Tarabusi, E., Figá, A., Talamanca, A.: Stable laws arising from hitting distributions of processes on homogeneous trees and the hyperbolic half-plane. Pacific J. Math. 197, 257-273 (2001)

[4] Baldi, P., Casadio Tarabusi, E., Figá, A., Talamanca, A., Yor, M.: Nonsymmetric hitting distributions on the hyperbolic half-plane and subordinated perpetuities. Rev. Mat. Iberoam. 17, 587-605 (2001)

[5] Brelot, M.: Axiomatique des Fonctions Harmonique. Presses Univ., Montréal (1966)

[6] Bogus, K., Byczkowski, T., Małeck, J.: Sharp estimates of the Green function of hyperbolic Brownian motion. Studia Mathematica 3, 197-121 (2015)

[7] Byczkowski, T., Graczyk, P., Stos, A.: Poisson kernels of half-spaces in real hyperbolic spaces. Rev. Mat. Iberoam. 23(1), 85-126 (2007)

[8] Byczkowski, T., Małecki, J.: Poisson kernel and Green function of the ball in real hyperbolic spaces. Potential Anal. 27(1), 1-26 (2007)

[9] Byczkowski, T., Ryznar, M.: Hitting distribution of geometric Brownian motion. Studia Math. 173, 19-38 (2006)

[10] Constantinescu, C., Cornea, A.: Potential Theory on Harmonic Spaces. Spinger, Berlin (1972)

[11] Eriksson, S.-L., Leutwiler, H.: Introduction to hyperbolic function theory. In: Proceedings of Clifford Algebras and Inverse Problems, Tampere University of Technology, Department of Mathematics, Research Report 90, pp. 1-28 (2009)

[12] Eriksson, S.-L., Orelma, H.: General integral formulas for k-hyper-mono-genic functions. Adv. Appl. Clifford Algebras 27(1), 99-110 (2017). https://doi.org/ 10.1007/s00006-015-0629-7

[13] Eriksson, S.-L., Vuojamo, V.: Integral kernels for k-hypermonogenic functions. Complex Var. Elliptic Equ. 62(9), 1254-1265 (2017). https://doi.org/10.1080/ 17476933.2016.1250402

[14] Franchi, J., Le Jan, Y.: Hyperbolic Dynamics and Brownian Motion. Oxford Mathematical Monographs, Oxford (2012)

[15] Gruet, J.-C.: Semi-groupe du mouvement Brownien hyperbolique. Stoch. Stoch. Rep. 56, 53-61 (1996)

[16] Gradshteyn, I.S., Ryzhik, I.M.: Table of Integrals, Series, and Products, 7th edn. Elsevier, Amsterdam (2007)

[17] Helms, L.: Introduction to Potential Theory. Wiley, New York (1969)

[18] Kakutani, S.: On Brownian motions in n-space. Proc. Imp. Acad. Tokyo 20, 648-652 (1944)

[19] Knapp, A.: Connection between Brownian motion and potential theory. J. Math. Anal. Appl. 12, 328-349 (1965)

[20] Leutwiler, H.: Best constants in the Harnack inequality for the Weinstein equation. Aequation Math. 34, 304-315 (1987) 
[21] Leutwiler, H.: Modified Clifford analysis. Complex Var. 17, 153-171 (1992)

[22] Leutwiler, H.: Appendix: Lecture notes of the course "Hyperbolic harmonic functions and their function theory". Clifford algebras and potential theory, 85109, Univ. Joensuu Dept. Math. Rep. Ser., 7, Univ. Joensuu, Joensuu (2004)

[23] Małecki, J., Serafin, G.: Hitting hyperbolic half-space. Demonstratio Mathematica 45(2), 337-360 (2012)

Sirkka-Liisa Eriksson

Department of Mathematics and Statistics

University of Helsinki

P.O. Box 6800014 Helsinki

Finland

e-mail: sirkka-liisa.eriksson@helsinki.fi

Terhi Kaarakka

Faculty of Information Technology and Communication Sciences

Computing Sciences

Tampere University

P.O. Box 55333101 Tampere

Finland

e-mail: terhi.kaarakka@tuni.fi

Received: January 28, 2020.

Accepted: October 10, 2020. 